\title{
ICD shock below the detection rate therapy zone. When appropriate is inadequate.
}

Victor García-Hernando ${ }^{1}$, Francisco Méndez Zurita ${ }^{1}$, Enrique Rodríguez-Font ${ }^{1}$, Concepcion Alonso-Martin ${ }^{1}$, Jose M Guerra ${ }^{2}$, Bieito Campos García ${ }^{1}$, Zoraida Moreno Weidmann , Alba Maestro Benedicto ${ }^{1}$, and Xavier Viñolas ${ }^{1}$

${ }^{1}$ Hospital de la Santa Creu i Sant Pau

${ }^{2}$ Hospital de la Santa Creu i Sant Pau, Universitat Autònoma de Barcelona, Institut de Recerca HSCSP-IIB Sant Pau.

September 24, 2021

\section{Hosted file}

VFTA final JCE.docx available at https://authorea.com/users/435720/articles/538546-icd-shockbelow-the-detection-rate-therapy-zone-when-appropriate-is-inadequate

\section{Hosted file}

Figures.pdf available at https://authorea.com/users/435720/articles/538546-icd-shock-belowthe-detection-rate-therapy-zone-when-appropriate-is-inadequate 